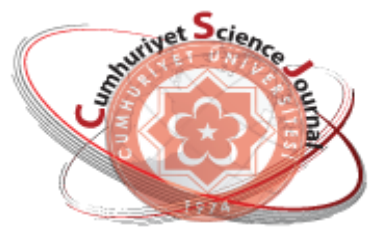

e-ISSN: 2587-246X

ISSN: $2587-2680$

\title{
Uniform and Pointwise Polynomial Estimates in Regions with Interior and Exterior Cusps
}

Pelin OZKARTEPE

University of Gaziantep, Faculty of Nizip Education, Gaziantep, TURKEY

\begin{abstract}
In this work, we investigate the estimation for algebraic polynomials in the bounded and unbounded regions with piecewise Dini smooth curve having interior and exterior zero angles.
\end{abstract}

Keywords: Algebraic polynomials

\section{İç ve Dış Sıfır Açılı Bölgelerde Polinom için Düzgün ve Noktasal Değerlendirmeler}

\footnotetext{
Özet: Bu çalışmada, içte ve dışta sıfır açı olan parçalı Dini düzgün eğri ile sınırlı sonlu ve sonsuz bölgelerdeki cebirsel polinomlar için düzgün ve noktasal değerlendirmeler inceledik.

Anahtar Kelimeler: Cebirsel polinomlar
}

\section{INTRODUCTION and MAIN RESULTS}

Let $\mathbb{C}$ be a complex plane and $\overline{\mathbb{C}}:=\mathbb{C} \cup\{\infty\} ; G \subset \mathbb{C}$ be the bounded Jordan region, with $0 \in G$ and the boundary $L:=\partial G$ be a closed Jordan curve, $\Omega:=\overline{\mathbb{C}} \backslash \bar{G}=\operatorname{ext} L . \Delta:=\{w:|w|>1\}$ (with respect to $\overline{\mathbb{C}}$ ). Let function $w=\Phi(z)$ be the univalent conformal mapping of $\Omega$ onto the $\Delta$ normalized by $\Phi(\infty)=\infty, \lim _{z \rightarrow \infty} \frac{\Phi(z)}{z}>0$ and $\Psi:=\Phi^{-1}$.

For $R>1$ let us set $L_{R}:=\{z:|\Phi(z)|=R\}, G_{R}:=\operatorname{int} L_{R}, \Omega_{R}:=\operatorname{ext} L_{R}$. For $z \in \mathbb{C}$ and $M \subset \mathbb{C}$ we set: $d(z, M)=\operatorname{dist}(z, M):=\inf \{|z-\zeta|: \zeta \in M\}$.

Let $\left\{z_{j}\right\}_{j=1}^{m} \in L$ be a fixed system of distinct points. Consider a so-called generalized Jacobi weight function $h(z)$ being defined as follows:

\footnotetext{
* Corresponding author. Email address: pelinozkartepe@gmail.com http://dergipark.gov.tr/csj @2016 Faculty of Science, Cumhuriyet University
} 


$$
h(z):=\prod_{j=1}^{m}\left|z-z_{j}\right|^{\gamma_{j}}, \quad z \in G_{R_{0}}, R_{0}>1
$$

where $\gamma_{j}>-1$ for every $j=1,2, \ldots, m$.

Denote by $\wp_{n}$ the class of all complex algebraic polynomials $P_{n}(z)$ of degree at most $n \in \mathbb{N}$.

For any $p>0$ we introduce:

$$
\begin{aligned}
&\left\|P_{n}\right\|_{p}:=\left\|P_{n}\right\|_{\mathfrak{J}_{p}(h, L)}:=\left(\int_{L} h(z)\left|P_{n}(z)\right|^{p}|d z|\right)^{1 / p}, 0<p<\infty ; \\
&\left\|P_{n}\right\|_{\infty}:=\left\|P_{n}\right\|_{\mathfrak{I}_{\infty}(1, L)}:=\max _{z \in G}\left|P_{n}(z)\right|, p=\infty .
\end{aligned}
$$

To evaluate $\left|P_{n}(z)\right|$ on the whole complex plane we proceed as follows: taking $\mathbb{C}=\bar{G} \cup \Omega$, we divide the problem into following two problems: find estimates

$$
\begin{aligned}
& \text { a) }\left|P_{n}(z)\right| \leq c . \mu_{n}(L, h, p)\left\|P_{n}\right\|_{p}, z \in \bar{G}, p>0 \\
& \text { b) }\left|P_{n}(z)\right| \leq c . \eta_{n}(L, h, d(z, L), p)\left\|P_{n}\right\|_{p}|\Phi(z)|^{n+1}, z \in \Omega, p>0,
\end{aligned}
$$

where $c=c(L, p)>0$ is a constant independent from $n, z, P_{n}$, and $\mu_{n}(L, h, p) \rightarrow \infty, \quad \eta_{n}(L, h, d(z, L), p) \rightarrow \infty$ (in general!) as $n \rightarrow \infty$, depending on the geometrical properties of curve $L$, weight function $h$ and parameter $p$.

We note that, the first results of type (1.2), in case $h(z) \equiv 1$ for $L=\{z:|z|=1\}$ and $0<p<\infty$ was found by Jackson [14]. The another results, similar to (1.2), for the sufficiently smooth curve, was obtained in [28] $(h(z) \equiv 1)$, and in [25, Part 4] $(h(z) \neq 1)$. The estimation of (1.2)-type for $0<p<\infty$ and $h(z) \equiv 1$ when $L$ is a rectifiable Jordan curve was investigated in [25-27], [16, 17], [19. pp.122-133], [21] obtained identical inequalities for more general curves and for another weighed function. There are more references regarding the inequality of (1.2)-type, we can find in Milovanovic et al. [18, Sect.5.3].

Further, analogous estimates of (1.2) for some regions and the weight function $h(z)$ were obtained: in [2] $(p>1)$ and in [20] $\left(p>0, h \equiv h_{0}\right)$ for regions bounded by rectifiable quasiconformal curve having some general properties; in [3] ( $p>1$ ) for piecewise Dini-smooth curve with interior and exterior cusps; in [4] $p>1$ for regions bounded by piecewise smooth curve with exterior cusps but without interior cusps; in [5] $p>0$ for regions bounded by piecewise rectifiable quasiconformal curve with cusps; in [6] $p>0$ for regions bounded by piecewise quasismooth (by Lavrentiev) curve with cusps.

The results of the (1.3) type starts from the work of Bernstein [29]. Analogous results of (1.3)-type for some norms and for different unbounded regions were obtained by Lebedev, Tamrazov, Dzjadyk (see, for example, [12, pp.418-428.]), Stylianopoulos [24] and others. Corresponding results (1.3) for some regions and the weight function $h(z)$ defined as in (1.1) with $\gamma_{j}>-1$ were also obtained: in [3] for $p>1$ and for regions bounded by piecewise Dini-smooth boundary with interior and exterior zero angles; in [6] for $p>0$ and for regions bounded by piecewise quasiconformal boundary with interior and exterior zero 
angles; in [4] for $p>1$ and for regions bounded by piecewise smooth boundary with exterior zero angles (without interior zero angles); in [6] for $p>0$ and for regions bounded by piecewise quasismooth boundary with interior and exterior zero angles and in others.

In this work, we investigate similar problem [3] for $0<p \leq 1$ in bounded $\bar{G}$ and unbounded region $\Omega$ with piecewise Dini-smooth curve having interior and exterior angles (also cusps) for weight function $h$ defined in (1.1) . Finally, combining obtained estimates for $\left|P_{n}(z)\right|$ on $\bar{G}$ and $\Omega$, we get the evaluation for $\left|P_{n}(z)\right|$ in whole complex plane, depending on the geometrical properties of the region $G$, weight function $h(z)$ and $p$.

\section{MAIN RESULTS}

Let us give some definitions and notations that will be used later in the text. In what follows, we always assume that $p>0$ and the constants $c, c_{0}, c_{1}, c_{2}, \ldots$ are positive and constants $\varepsilon_{0}, \varepsilon_{1}, \varepsilon_{2}, \ldots$ are sufficiently small positive (generally, are different in different relations), which depends on $G$ in general and, on parameters inessential for the argument, otherwise, the dependence will be explicitly stated. Also note that, for any $k \geq 0$ and $m>k$, notation $j=\overline{k, m}$ denotes $j=k, k+1, \ldots, m$. Let us give some definitions and notations that will be used later in the text.

Let $S$ be a rectifiable Jordan curve or arc and $z=z(s), s \in[0,|S|],|S|:=$ mes $S$, denote the natural representation of $S$.

Definition 2.1.[22, p.48](see also [11, p.32]) We say that a Jordan curve or arc S called Dinismooth, if it has a parametrization $z=z(s), 0 \leq s \leq|S|$, such that $z^{\prime}(s) \neq 0,0 \leq s \leq|S|$ and $\left|z^{\prime}\left(s_{2}\right)-z^{\prime}\left(s_{1}\right)\right|<g\left(s_{2}-s_{1}\right), s_{1}<s_{2}$, where $g$ is an increasing function for which

$$
\int_{0}^{1} \frac{g(x)}{x} d x<\infty .
$$

Now, we will define a new class of regions with piecewise Dini-smooth boundary, which have at the boundary points corners, interior and exterior cusps simultaneously. Let $C_{+}^{2}[0,1]$ denote the class of all functions $f:\left[0, \varepsilon_{0}\right] \rightarrow \mathbb{R}$ which are twice differentiable such that $f(0)=0$ and $f^{(k)}(x)>0$ for all $0<x \leq \varepsilon_{0}, k=0,1,2$.

Definition 2.2. We say that a Jordan region $G \in P D S\left(\lambda_{1}, \ldots, \lambda_{m_{1}} ; f_{m_{1}+1}, \ldots, f_{m}\right), 0<\lambda_{i} \leq 2 i=\overline{1, m}_{1}$, $f_{j} \in C_{+}^{2}[0,1], j=\overline{m_{1}+1, m}$, if $L=\partial G$ consists a union of finite number of Dini-smooth $\operatorname{arcs}\left\{L_{j}\right\}_{j=0}^{m}$, joining at the points $\left\{z_{j}\right\}_{j=0}^{m} \in L$ such that Lis locally Dini-smooth at $z_{0}$ and the following properties hold:

1) for all $i=\overline{1, m_{1}}$ the arcs $L_{i-1}$ and $L_{i}$ meet at the point $z_{i}$ with the exterior (respect to $\bar{G}$ ) angle $\lambda_{i} \pi$, $0<\lambda_{i} \leq 2$ 
2) for all $j=\overline{m_{1}+1, m}$, the arcs $L_{j-1}$ and $L_{j}$ meet at the point $z_{j}$ with $f_{j}$-type exterior zero angle, that is there exists an $\varepsilon_{j}$-neighborhood of $z_{j}$ such that in a local coordinate system, with the origin at $z_{j}$, we have

$$
\left\{z=x+i y:|z|<\varepsilon_{j}, c_{1, j} f_{j}(x) \leq y \leq c_{2, j} f_{j}(x), x \in\left[0, \varepsilon_{j}\right]\right\} \subset \bar{\Omega}
$$

and

$$
\left\{z=x+i y:|z|<\varepsilon_{j},|y| \geq \varepsilon_{0} x, x \in\left[0, \varepsilon_{j}\right]\right\} \subset \bar{G}
$$

for some constants $-\infty<c_{1, j}<c_{2, j}<\infty$ and $\varepsilon_{0}, \varepsilon_{j}$.

Throughout this work, we will assume that the points $\left\{z_{j}\right\}_{j=1}^{m} \in L$ defined in (1.1) and Definition 2.2. are the same. Without loss of generality, we assume that these points on the curve $L=\partial G$ are located in the positive direction such that, $G$ has $\lambda_{j} \pi, 0<\lambda_{j} \leq 2, j=\overline{1, m_{1}}$, exterior angles (when $\lambda_{j}=2-$ interior zero angles (interior cusps)) at the points $\left\{z_{j}\right\}_{j=1}^{m_{1}}, m_{1} \leq m$, and has exterior zero angles (exterior cusps) on the points $\left\{z_{j}\right\}_{j=m_{1}+1}^{m}$ and $w_{j}:=\Phi\left(z_{j}\right)$

For the simplicity of exposition and in order to avoid cumbersome calculations, without loss of generality, we consider, a Jacobi weight function $h$ defined by (1.1) and the region $G \in P D S\left(\lambda_{1} ; f_{2}\right)$ with $m_{1}=1, m=2,0<\lambda_{1} \leq 2$ and the function $f_{2}(x)=x^{1+\alpha_{2}}, \alpha_{2}>0$, as the function $f_{2}$ in the Definition 2.2. We will use the notation $G \in P D S\left(\lambda_{1} ; f_{2}\right)$ for this construction. Therefore, $G \in P D S\left(\lambda_{1} ; f_{2}\right)$ denote that the region $G$ may have exterior $\lambda_{1} \pi, 0<\lambda_{1} \leq 2$, (also interior zero) angle at the point $z_{1}$ and exterior zero angle at the point $z_{2}$ of $f_{2}(x)=x^{1+\alpha_{2}}$-touching. Correspondingly, we will use the notation $G \in P D S\left(\lambda_{1}, \lambda_{2}\right)$, if $m_{1}=m=2$, i.e., the region $G$ may have only exterior $\lambda_{i} \pi, 0<\lambda_{i} \leq 2$, (also interior zero) angles at the point $z_{i}, i=1,2$, without exterior zero angles, and notation $G \in \operatorname{PDS}\left(f_{1}, f_{2}\right)$, if $m_{1}=0, m=2$, i.e., the region $G$ may have only exterior zero angles of $f_{j}(x)=x^{1+\alpha_{j}}-$ touching at the point $z_{j}, j=1,2$, without exterior (also interior zero) angles at the point $z_{j}$. When $m_{1}=m=0$, this definition yields a Jordan region whose boundary is a Dini-smooth curve.

Now, we can state our new results. We introduce some notation, which we use in what follows:

$$
\begin{gathered}
\lambda^{*}\left\{\begin{array}{ccc}
\max \left\{\lambda_{i}, 1\right\} & \text { if } & 0<\lambda_{i}<2, \\
2 & \text { if } & \lambda_{i}=2,
\end{array}\right. \\
\gamma_{i}^{*}:=\max \left\{\gamma_{i}, 0\right\}, \lambda_{i, \alpha_{i}}^{* *}:=\max \left\{\gamma_{i} ;-\alpha_{i}\right\}, i=1,2 ; \\
\lambda^{*}=\max \left\{\lambda_{1}^{*}, \lambda_{2}^{*}\right\}, \gamma^{*}=\max \left\{\gamma_{1}^{*}, \gamma_{2}^{*}\right\}, \gamma_{\alpha}^{*}:=\max \left\{\gamma_{2, \alpha_{i}}^{*}, \gamma_{2, \alpha_{2}}^{*}\right\} \\
\alpha^{*}:=\max \left\{\alpha_{1}, \alpha_{2}\right\}, \alpha_{*}:=\min \left\{\alpha_{1}, \alpha_{2}\right\}
\end{gathered}
$$


Theorem 2.1. Let $G \in \operatorname{PDS}\left(\lambda_{1} ; f_{2}\right)$ for some $0<\lambda_{1} \leq 2$ and $f_{2}(x)=c x^{1+\alpha_{2}}, \alpha_{2}>0 ; h(z)$ be defined as in (1.1). Then, for any $P_{n} \in \wp_{n}, n \in \mathbb{N}$ and $\gamma_{j}>-1, j=1,2$, we have:

$$
\left\|P_{n}\right\|_{\infty} \leq c_{1} A_{n}\left\|P_{n}\right\|_{p}
$$

where $c_{1}=c_{1}\left(G, \gamma_{j}, \lambda_{1}, \alpha_{2}, p\right)>0$ is the constant, independent from $z$ and $n$;

$$
A_{n}:=\left\{\begin{array}{ccc}
n^{\frac{\gamma_{1}^{*}+1}{p} \lambda_{1}^{*}}, & \gamma_{1}>-1, & -1<\gamma_{2}<-\alpha_{2}, \\
n^{\frac{\gamma_{2}+1+2 \alpha_{2}}{p\left(1+\alpha_{2}\right)}}, & -1<\gamma_{1}<\frac{\gamma_{2}+1+2 \alpha_{2}}{\lambda_{1}}-1, & \gamma_{2} \geq-\alpha_{2}, \\
n^{\frac{\gamma_{1}+1}{p} \lambda_{1}^{*}}, & \gamma_{1} \geq \frac{\gamma_{2}+1+2 \alpha_{2}}{\lambda_{1}\left(1+\alpha_{2}\right)}-1, & \gamma_{2} \geq-\alpha_{2},
\end{array}\right.
$$

and $\tilde{\gamma}_{1}, \lambda_{1}$ are defined as in (2.1).

Now, let's take that the curve $L$ in the both points $z_{1}, z_{2} \in L$ have exterior non zero angles. In this case we obtain:

Theorem 2.2. Let $G \in P D S\left(\lambda_{1}, \lambda_{2}\right)$ for some $0<\lambda_{1}, \lambda_{2} \leq 2 ; h(z)$ be defined as in (1.1). Then, for any $P_{n} \in \wp_{n}, n \in \mathrm{N}, \gamma_{i}>-1, i=1,2$, we have:

$$
\left\|P_{n}\right\|_{\infty} \leq c_{2} n^{\frac{\gamma^{*}+1}{p} \lambda^{*}}\left\|P_{n}\right\|_{p}
$$

where $c_{2}=c_{2}\left(G, \gamma_{i}, \lambda_{i}, p\right)>0$ is the constant, independent from $z$ and $n$, and $\gamma^{*}, \lambda^{*}$ are defined as in (2.1). Analogously, when the curve $L$ in the both points $z_{1}, z_{2} \in L$ have only exterior zero angles, we have:

Theorem 2.3. Let $G \in \operatorname{PDS}\left(f_{1}, f_{2}\right)$ for some $f_{j}(x)=c x^{1+\alpha_{j}}, \alpha_{j}>0, j=1,2 ; h(z)$ be defined as in (1.1). Then, for any $P_{n} \in \wp_{n}, n \in \mathbb{N}, \gamma_{j}>-1, j=1,2$, we have:

$$
\left\|P_{n}\right\|_{\infty} \leq c_{3} n^{\frac{\gamma^{*} \alpha^{+1}}{p+1+\alpha_{*}}+\frac{2 \alpha^{*}}{p\left(1+\alpha^{*}\right)}}\left\|P_{n}\right\|_{p}
$$

where $c_{3}=c_{3}\left(G, \gamma_{j}, \alpha_{j}, p\right)>0$ is the constant, independent from $z$ and $n$, and $\gamma_{\alpha}^{*}, \alpha^{*}, \alpha_{*}$ are defined as in (2.1).

Now, we will estimate of $\left|P_{n}(z)\right|$ at the critical points $z_{j} \in L, j=1,2$.

Theorem 2.4. Let $G \in P D S\left(\lambda_{1} ; f_{2}\right)$ for some $0<\lambda_{1} \leq 2$ and $f_{2}(x)=c x^{1+\alpha_{2}}, \alpha_{2}>0 ; h(z)$ be defined as in (1.1). Then, for any $P_{n} \in \wp_{n}, n \in \mathrm{N}, \gamma_{j}>-1, j=1,2$, we have: 


$$
\left|P_{n}\left(z_{j}\right)\right| \leq c_{4} B_{n}\left\|P_{n}\right\|_{p},
$$

where $c_{4}=c_{4}\left(G, \gamma_{j}, \lambda_{1}, \alpha_{2}, p\right)>0$ is the constant, independent from $z$ and $n$;

$$
B_{n}:= \begin{cases}n^{\frac{\gamma_{1}+1}{p} \lambda_{1}^{*}}, & \text { for } j=1, \\ n^{\frac{\gamma_{2, \alpha_{2}+1+2 \alpha_{2}}}{p\left(1+\alpha_{2}\right)},} & \text { f or } j=2 .\end{cases}
$$

Remark 2.1. The similar results from [2] (for $p>1$ ); [5] (for $p>0$ ); [6] (for $p>0$ ) were obtained for regions of a more general geometric configuration, but without exterior non-zero angles in the usual sense. Therefore, the estimates were obtained in Theorems 2.1-2.4 can be compared with correspondingly results from [4]. The results obtained in Theorems 2.1-2.4 extend the results obtained in [4] in case $0<p<1$ : In Theorems 2.1-2.4 we also shown that by slightly increasing the smoothness of the boundary, one can achieve improvement in growth by $n^{\varepsilon}$ for arbitrary small $\varepsilon>0$ :

Remark 2.2. Let us give a geometric interpretation of Theorem 2.4. Obviously, $\operatorname{PDS}\left(\lambda_{1} ; f_{2}\right) \subset \operatorname{PDS}\left(\lambda_{2} ; f_{2}\right)$ for $0<\lambda_{1}<\lambda_{2} \leq 2$, and for the same fixed $f_{2}=c x^{1+\alpha_{2}} ; \alpha_{2}>0$. Therefore, if $\lambda_{1}<\lambda_{2}$, then the region $G\left(\lambda_{1}\right) \subset P D S\left(\lambda_{1} ; f_{2}\right)$ also have a $G\left(\lambda_{1}\right) \subset \operatorname{PDS}\left(\lambda_{2} ; f_{2}\right)$. If $\lambda_{1}<\lambda_{2}$, then the region $G\left(\lambda_{1}\right)$ has a more obtuse angle at the point $z_{1}$ than the region $G\left(\lambda_{2}\right)$. Hence, the sharper from the inside angle at the point $z_{1}$ , the worse the degree of $n$ and the obtuse from the inside the angle at the point $z$, the better the degree of $n$.

The same can be said for another point $z_{2}$. In this case, we see that $\operatorname{PDS}\left(\lambda_{1} ; f_{2}\right) \subset \operatorname{PDS}\left(\lambda_{2} ; \mathrm{g}_{2}\right)$ for $f_{2}=c x^{1+\alpha_{2}} ; g_{2}=c x^{1+\beta_{2}}, 0<\alpha_{2}<\beta_{2}$, and for the same fixed $\lambda_{1}, 0<\lambda_{1} \leq 2$. Hence, the sharper the exterior angle at the point $z_{2}$, the worse the degree of $n$ and the obtuse the exterior angle at the point $z_{2}$, the better the degree of $n$ for some $\gamma_{2}<1$.

Now, we will give pointwise estimations for $\left|P_{n}(z)\right|$ in regions $G \in P D S\left(\lambda_{1} ; f_{2}\right)$, at the $z \in \Omega$.

Theorem 2.5. Let $G \in \operatorname{PDS}\left(\lambda_{1} ; f_{2}\right)$ for some $0<\lambda_{1} \leq 2$ and $f_{2}(x)=c x^{1+\alpha_{2}}, \alpha_{2}>0 ; h(z)$ be defined as in (1.1). Then, for any $P_{n} \in \wp_{n}, n \in \mathrm{N}$, and $\gamma_{j}>-1, j=1,2$, we have:

$$
\left|P_{n}(z)\right| \leq c_{5} \frac{D_{n}}{d^{p / 2}(z, L)}\left\|P_{n}\right\|_{p}|\Phi(z)|^{n+1}, z \in \Omega
$$

where $c_{5}=c_{5}\left(G, \gamma_{j}, \lambda_{1}, \alpha_{2}, p\right)>0$ is the constant, independent from $z$ and $n$;

$$
D_{n}:=\left\{\begin{array}{cc}
n^{\frac{(1-1) \lambda_{1}^{*}}{p}}+n^{\frac{\gamma_{2}-1}{p\left(1+\alpha_{2}\right)}}, & \gamma_{1}, \gamma_{2}>1, \\
(\ln n)^{\frac{1}{p}}, & \gamma_{1} \leq \gamma_{2}=1, \\
1, & -1<\gamma_{1}, \gamma_{2}<1 .
\end{array}\right.
$$


Combining Theorems 2.5 and 2.1, we can obtain the following estimate for $\left|P_{n}(z)\right|$ in the whole complex plane.

Corollary 2.1. Under the assumptions of the Theorem 2.5, we have:

$$
\left|P_{n}(z)\right| \leq c_{6}\left\|P_{n}\right\|_{p}\left\{\begin{array}{cc}
A_{n}, & z \in \bar{G}, \\
\frac{|\Phi(z)|^{n+1}}{d^{\frac{2}{p}}(z, L)} D_{n}, & z \in \Omega,
\end{array}\right.
$$

where $c_{6}=c_{6}\left(G, \gamma_{j}, \lambda_{1}, \alpha_{2}, p\right)>0$ is the constant, independent from $z$ and $n$; and $A_{n}$ and $D_{n}$ are defines as in (2.3) and (2.8) correspondingly.

\subsection{Sharpness of estimates}

The sharpness of the estimations (2.2)-(2.7) for some special cases can be discussed by comparing them with the following results:

Remark 2.1. For any $n \in \mathbb{N}$ and $i=1,2,3$ there exist polynomials $P_{i, n} \in \wp_{n}$, regions $G^{i} \subset \mathbb{C}$ and constants $c_{7}=c_{7}\left(G^{1}\right)>0, c_{8}=c_{8}\left(G^{1}\right)>0$, and $c_{9}=c_{9}\left(G^{2}\right)>0$ such that:

$$
\begin{aligned}
& \left\|P_{1, n}\right\|_{C\left(\overline{G^{1}}\right)} \geq c_{7} n^{\frac{1}{p}}\left\|P_{1, n}\right\|_{\mathfrak{I}_{p}\left(\widehat{\partial G} G^{1}\right)}, \\
& \left\|P_{2, n}\right\|_{C\left(\overline{G^{1}}\right)} \geq c_{8} n^{\left(\gamma_{1}+1\right) / p}\left\|P_{2, n}\right\|_{\mathfrak{I}_{p}\left(|z-1|^{p^{1}}, \partial G^{1}\right)}, \gamma_{1}>0, p>\gamma_{1}+1,
\end{aligned}
$$

and

$$
\left|P_{3, n}(z)\right| \geq c_{9}|\Phi(z)|^{n}\left\|P_{3, n}\right\|_{\mathfrak{J}_{2}\left(\partial G^{2}\right)}, \forall z \in F
$$

where $F$ is a closed subset in $\bar{\Omega} \backslash \overline{G^{2}}$.

\section{SOME AUXILIARY RESULTS}

For the nonnegative functions $a>0$ and $b>0$, we shall use the notations $a \prec b$ (order inequality), if $a \leq c b$ and $a \approx b$ are equivalent to $c_{1} a \leq b \leq c_{2} a$ for some constants $c, c_{1}, c_{2}$ (independent of $a$ and $b$ ) respectively.

We can find a well known definition of a $K$-quasiconformal curve in [7], [15, p.97], [22,p.286] and [23] as follows:

Definition 3.1.[15, p.97], [23]The Jordan curve (or arc) Lis called $K$ - quasiconformal ( $K \geq 1$ ), if there is a $K$-quasiconformal mapping $f$ of the region $D \supset L$ such that $f(L)$ is a circle (or line segment). 
Let $F(L)$ denote the set of all sense preserving plane homeomorphisms $f$ of the region $D \supset L$ such that $f(L)$ is a line segment (or circle) and let

$$
K_{L}:=\inf \{K(f): f \in F(L)\}
$$

where $K(f)$ is the maximal dilatation of a such mapping $f$. Then $L$ is a quasiconformal curve, if $K_{L}<\infty$, and $L$ is a $K$ - quasiconformal curve, if $K_{L} \leq K$.

We well know that there exist quasiconformal curves which are not rectifiable [15, p.104]. According to the "three-point" criterion [6], every piecewise Dini-smooth curve (without any cusps) is quasiconformal.

Lemma 3.1. [1] Let Lbe a $K$-quasiconformal curve, $z_{1} \in L, z_{2}, z_{3} \in \Omega \cap\left\{z:\left|z-z_{1}\right| ? d\left(z_{1}, L_{r_{0}}\right)\right\}$; $w_{j}=\Phi\left(z_{j}\right), j=1,2,3$. Then

a)The statements $\left|z_{1}-z_{2}\right| \prec\left|z_{1}-z_{3}\right|$ and $\left|w_{1}-w_{2}\right| \prec\left|w_{1}-w_{3}\right|$ are equivalent.

So statements $\left|z_{1}-z_{2}\right| \approx\left|z_{1}-z_{3}\right|$ and $\left|w_{1}-w_{2}\right| \approx\left|w_{1}-w_{3}\right|$ also equivalent; b) If $\left|z_{1}-z_{2}\right| \prec\left|z_{1}-z_{3}\right|$, then

$$
\left|\frac{w_{1}-w_{3}}{w_{1}-w_{2}}\right|^{K^{2}} \prec\left|\frac{z_{1}-z_{3}}{z_{1}-z_{2}}\right| \prec\left|\frac{w_{1}-w_{3}}{w_{1}-w_{2}}\right|^{K^{-2}},
$$

where $0<r_{0}<1$, is constant, depending on $G$.

Recall that for $\quad 0<\delta_{j}<\delta_{0}:=\frac{1}{4} \min \left\{\left|z_{i}-z_{j}\right|: i, j=1,2, \ldots, m, i \neq j\right\}$, we put $\Omega\left(z_{j}, \delta_{j}\right):=\Omega \cap\left\{z:\left|z-z_{j}\right| \leq \delta_{j}\right\} ; \delta:=\min _{1 \leq j \leq m} \delta_{j}, \Omega(\delta):=\bigcup_{j=1}^{m} \Omega\left(z_{j}, \delta\right), \Omega:=\Omega \backslash \Omega(\delta)$. Additionally, let $\Delta_{j}:=\Phi\left(\Omega\left(z_{j}, \delta\right)\right), \Delta(\delta):=\bigcup_{j=1}^{m} \Phi\left(\Omega\left(z_{j}, \delta\right)\right), \Delta(\delta):=\Delta \backslash \Delta(\delta)$.

The following lemma is a consequence of the results given in [22, pp.41-58], [11, pp.32-36], and estimation for the $\left|\Psi^{\prime}\right|$ (see, for example, [10, Th.2.8]):

$$
\left|\Psi^{\prime}(\tau)\right| \approx \frac{d(\Psi(\tau), L)}{|\tau|-1} .
$$

Lemma 3.2. Let a Jordan region $G \in P D S\left(\lambda_{j} ; 0\right), 0<\lambda_{j} \leq 2, j=\overline{1, m_{1}}$. Then,

i) for any $w \in \Delta_{j},\left|\Psi(w)-\Psi\left(w_{j}\right)\right| \approx\left|w-w_{j}\right|^{\lambda_{j}},\left|\Psi^{\prime}(w)\right| \approx\left|w-w_{j}\right|^{\lambda_{j}-1}$; 
ii) for any $w \in \bar{\Delta} \backslash \Delta_{j}\left|\Psi(w)-\Psi\left(w_{j}\right)\right| \approx\left|w-w_{j}\right|,\left|\Psi^{\prime}(w)\right| \approx 1$.

Let $\left\{z_{j}\right\}_{j=1}^{m}$ be a fixed system of the points on $L$ and the weight function $h(z)$ defined as (1.1).

Lemma 3.3. [3] Let $L$ be a rectifiable Jordan curve; $h(z)$ as defined in (1.1). Then, for arbitrary $P_{n}(z) \in \wp_{n}$, any $R>1$ and $n \in \mathbb{N}$ we have

$$
\left\|P_{n}\right\|_{\mathfrak{I}_{p}\left(h, L_{R}\right)} \leq R^{n+\frac{1+\gamma^{* *}}{p}}\left\|P_{n}\right\|_{\mathfrak{I}_{p}(h, L)}, p>0
$$

where $\gamma^{* *}=\max \left\{\gamma_{k}: \gamma_{k} \in \Gamma, k \leq m\right\}$.

\section{PROOF OF THEOREMS}

Before giving proofs of the main theorems, let us give the geometric notations used in the proofs to prevent the flow of presentation of the proof.

Without loss of generality, we assume that $z_{1}=-1, z_{2}=1$ and $(-1,1) \subset G$. We will use the notations given in the following:

$L^{+}:=\{z \in L: \quad \operatorname{Im} z \geq 0\}$ and $L^{-}:=\{z \in L: \operatorname{Im} z<0\}$, so that $L=L^{+} \cup L^{-} ; w_{j}=\Phi\left(z_{j}\right)=: e^{i \phi_{j}}$, $0 \leq \phi_{j}<2 \pi, j=1,2 ; w^{+}:=e^{i \theta_{+}}$and $w^{-}:=-w^{+}$, where $\theta_{+}=\phi_{1}+\frac{\left|\phi_{1}-\phi_{2}\right|}{2} ; z^{ \pm}:=\Psi\left(w^{ \pm}\right) ; l_{j}^{ \pm}:=l_{j}^{ \pm}\left(z_{i}, z^{ \pm}\right)$is the subarc with endpoints $z^{ \pm}$and $z_{j}, j=1,2, . L^{1}:=L^{1}\left(z^{+}, z_{1}, z^{-}\right)$denote the arc connecting the points $z^{+}$and $z^{-}$passing through the point $z_{1} ; L^{2}:=L^{2}\left(z^{-}, z_{2}, z^{+}\right)$denote the arc, connecting the points $z^{-}$and $z^{+}$passing through the point $z_{2}$.

Similar notations for $L_{R}$ are in the following: $L_{R}^{+}:=\left\{z \in L_{R}: \operatorname{Im} z \geq 0\right\}$ and $L_{R}^{-}:=\left\{z \in L_{R}: \quad \operatorname{Im} z<0\right\}$, so that $L_{R}=L_{R}^{+} \cup L_{R}^{-} ; w_{R}^{+}:=\operatorname{Re}^{i \theta_{+}}$and $w_{R}^{-}:=-w_{R}^{+} ; z_{R}^{ \pm}:=\Psi\left(w_{R}^{ \pm}\right)$. Let $z_{j, R} \in L_{R} \quad$ such that $d\left(z_{j}, L_{R}\right)=\left|z_{j}-z_{j, R}\right|, \quad j=1,2 ; \quad \zeta^{ \pm} \in L^{ \pm} \quad$ such that $d\left(z_{2, R}, L^{ \pm}\right)=\left|z_{2, R}-\zeta^{ \pm}\right|$. $z_{j}^{ \pm} \in L^{j} \cap L^{ \pm} \quad$ such that $c_{j} d\left(z_{j}, L_{R}\right)=\left|z_{j}-z_{j}^{ \pm}\right|, \quad j=1,2 ; \quad z_{j, R}^{ \pm} \in L_{R}^{ \pm} \quad$ such that $\quad c_{j} d\left(z_{j}, L_{R}\right)=\left|z_{j}-z_{j, R}^{ \pm}\right|$, $j=1,2$.

Finally, let us give the following notations: Let $E_{1, R}^{i, \pm}:=\left\{\zeta \in L_{R}^{ \pm}:\left|\zeta-z_{i}\right|<c_{i} d\left(z_{i}, L_{R}\right)\right\}$, and let $E_{2, R}^{i, \pm} \quad$ be the sub-arc of $L_{R}^{\phi} \quad$ with endpoints $z_{j, R}^{ \pm}$and $z_{R}^{ \pm} \quad, j=1,2$; $E_{1}^{i, \pm}:=\left\{\zeta \in L^{i}:\left|\zeta-z_{i}\right|<c_{i} d\left(z_{i}, L_{R}\right)\right\}$, and let $E_{2}^{i, \pm}$ be the sub-arc of $L^{ \pm}$with endpoints $z_{i}^{ \pm}$and $z^{ \pm}$, $i=1,2$. Let $F_{j, R}^{i, \pm}:=\Phi\left(E_{j, R}^{i, \pm}\right), i=1,2, j=1,2$. Let $z_{0} \in L$ be a point far from the points $z_{1}$ and $z_{2}$. Without loss of generality, we assume that $z_{0}=z^{+}$(or $z_{0}=z^{-}$) to ensure simplicity in calculations. 


\subsection{Proof of Theorem 2.1.}

Proof. Let $G \in \operatorname{PDS}\left(\lambda_{1} ; f_{2}\right)$ for some $0<\lambda_{1} \leq 2$ and $f_{2}(x)=c x^{1+\alpha_{2}}, \alpha_{2}>0$ and $R=1+\frac{1}{n}, n \in \mathbb{N}$. Let $w=\phi_{R}(z)$ denote the univalent conformal mapping of $G_{R}$ onto the unit disk $B=\{z:|z|<1\}$ normalized by $\phi_{R}(0)=0, \phi_{R}^{\prime}(0)>0$, and let $\left\{\xi_{j}\right\}, 1 \leq j \leq m \leq n$, the zeros of $P_{n}(z)$, lying on $G_{R}$ (if such zeros exist). Lets define a Blaschke function with respect to the zeros $\left\{\xi_{j}\right\}_{j=1}^{m}$ as follows:

$$
q_{m, R}(z)=\prod_{j=1}^{m} \frac{\phi_{R}(z)-\phi_{R}\left(\xi_{j}\right)}{1-\overline{\phi_{R}\left(\xi_{j}\right)} \phi_{R}(z)}
$$

and for any $p>0$ we put:

$$
Q_{n, p}(z):=\left[\frac{P_{n}(z)}{q_{m, R}(z)}\right]^{p / 2}, \quad z \in G_{R}
$$

The function $Q_{n, p}$ is analytic in $G_{R}$, continuous on $\bar{G}_{R}$ and does not have zeros in $G_{R}$. We take an arbitrary continuous branch of $Q_{n, p}$ and we maintain the same designation for this branch. Its Cauchy integral representation for the region $G_{R}$ is the following:

$$
Q_{n, p}(z)=\frac{1}{2 \pi i} \int_{L_{R}} Q_{n, p}(\zeta) \frac{d \zeta}{\zeta-z}, \quad z \in G_{R}
$$

From (4.1) and (4.2), we have:

$$
\left|P_{n}(z)\right|^{\frac{p}{2}} \leq\left|q_{m, R}(z)\right|^{\frac{p}{2}} \int_{L_{R}}\left|\frac{P_{n}(\zeta)}{q_{m, R}(\zeta)}\right|^{\frac{p}{2}} \frac{|d \zeta|}{|\zeta-z|} \leq \int_{L_{R}}\left|P_{n}(\zeta)\right|^{\frac{p}{2}} \frac{|d \zeta|}{|\zeta-z|},
$$

for all $z \in G_{R}$, since $\left|q_{m, R}(\zeta)\right| \equiv 1$ for all $\zeta \in L_{R}$ and $\left|q_{m, R}(z)\right|<1$ for all $z \in G_{R}$. Multiplying the numerator and the denominator of the last integrand by $h^{1 / 2}(\zeta)$, and then applying the Cauchy-Schwarz Inequality, we get:

$$
\left|P_{n}(z)\right|^{\frac{p}{2}} \leq\left(\int_{L_{R}} h(\zeta)\left|P_{n}(\zeta)\right|^{p}|d \zeta|\right)^{1 / 2}\left(\int_{L_{R}} \frac{|d \zeta|}{h(\zeta)|\zeta-z|^{2}}\right)^{1 / 2}
$$

According to the Lemma 3.3, we have:

$$
\left|P_{n}(z)\right| \leq\left\|P_{n}\right\|_{p}\left(\int_{L_{R}} \frac{|d \zeta|}{h(\zeta)|\zeta-z|^{2}}\right)^{1 / p}:=\left\|P_{n}\right\|_{p} J_{n}^{1 / p}
$$

for all $z \in G_{R}$, where 


$$
J_{n}:=J_{n}\left(L_{R}\right):=\int_{L_{R}} \frac{|d \zeta|}{h(\zeta)|\zeta-z|^{2}}
$$

Now, we will estimate the integral $J_{n}$. For this purpose, we will use the notations and definitions given in the beginning of this section. Under this notations, we can write $J_{n}$ in the following form:

$$
J_{n} \approx \sum_{i, j=1}^{2} \int_{E_{j, R}^{i, .}} \frac{|d \zeta|}{\left|\zeta-z_{i}\right|^{\gamma_{i}}|\zeta-z|^{2}}:=\sum_{i, j=1}^{2} J\left(E_{j, R}^{i, \pm}\right)
$$

Now, we estimate the integrals $J\left(E_{j, R}^{i, \pm}\right)$ in (4.5). First, let us fix a point $z^{\prime} \in L$, such that $\left|P_{n}\left(z^{\prime}\right)\right|=\left\|P_{n}\right\|_{C(\bar{G})}$. So that $z^{\prime} \in L^{1} \quad$ or $\quad z^{\prime} \in L^{2}$. We will examine both cases. Let $d_{i, R}:=d\left(z_{i}, L_{R}\right)$, $i=1,2$.

Case 1. Let $z^{\prime} \in L^{1}$. If $\gamma_{1} \geq 0$, we have:

$$
J\left(E_{1, R}^{1,+}\right)+J\left(E_{1, R}^{1,-}\right)=\int_{E_{1, R}^{1,+} \cup E_{1, R}^{1,-}} \frac{|d \zeta|}{\left|\zeta-z_{1}\right|^{\gamma_{1}}\left|\zeta-z^{\prime}\right|^{2}} \prec \frac{1}{d_{1, R}^{2}} \int_{d_{1, R}}^{c_{1} d_{1, R}} \frac{d s}{s^{\gamma_{1}}} \prec \frac{1}{d_{1, R}^{1+\gamma_{1}}},
$$

if $-1<\gamma_{1}<0$, then we have:

$$
J\left(E_{1, R}^{1,+}\right)+J\left(E_{1, R}^{1,-}\right) \prec \frac{1}{d_{1, R}^{2+\gamma_{1}}} \int_{d_{1, R}}^{c_{1} d_{1, R}} d s \prec \frac{1}{d_{1, R}^{1+\gamma_{1}}} .
$$

If $z^{\prime} \in E_{1}^{1, \pm}$, then we get:

$$
J\left(E_{2, R}^{1,+}\right)+J\left(E_{2, R}^{1,-}\right) \prec \int_{E_{2, R}^{1,+} \cup E_{2, R}^{1,-}} \frac{|d \zeta|}{\left(\min \left\{\left|\zeta-z_{1}\right|,\left|\zeta-z^{\prime}\right|\right\}\right)^{2+\gamma_{1}}} \prec \frac{1}{d_{1, R}^{1+\gamma_{1}}},
$$

for $\gamma_{1} \geq 0$, and taking into account the inequality $(x+y)^{r} \leq 2^{r}\left(x^{r}+x^{r}\right), \quad, x, y, r>0$, we have:

$$
\begin{aligned}
& J\left(E_{2, R}^{1,+}\right)+J\left(E_{2, R}^{1,-}\right)=\int_{E_{2, R}^{1,} \cup E_{2, R}^{1,-}} \frac{\left|\zeta-z_{1}\right|^{-\gamma_{1}}|d \zeta|}{\left|\zeta-z^{\prime}\right|^{2}} \\
\prec & \int_{E_{2, R}^{1,+} \cup E_{2, R}^{1,-}} \frac{\left|\zeta-z^{\prime}\right|^{-\gamma_{1}}+\left|z^{\prime}-z_{1}\right|^{-\gamma_{1}}}{\left|\zeta-z^{\prime}\right|^{2}}|d \zeta| \prec \int_{E_{2, R}^{1,+} \cup E_{2, R}^{1,-}} \frac{|d \zeta|}{\left|\zeta-z^{\prime}\right|^{2+\gamma_{1}}}+\left(c_{1} d_{1, R}\right)^{-\gamma_{1}} \\
\prec & \int_{E_{2, R}^{1,+} \cup E_{2, R}^{1,-}} \frac{|d \zeta|}{\left|\zeta-z^{\prime}\right|^{2}} \prec \frac{1}{d_{1, R}^{1+\gamma_{1}}} .
\end{aligned}
$$

for $-1<\gamma_{1}<0$. If $z^{\prime} \in E_{2}^{1, \pm}$, then we get: 


$$
J\left(E_{2, R}^{1,+}\right)+J\left(E_{2, R}^{1,-}\right)=\int_{E_{2, R}^{1,+} \cup E_{2, R}^{1,-,}} \frac{|d \zeta|}{\left|\zeta-z_{1}\right|^{\gamma_{1}}\left|\zeta-z^{\prime}\right|^{2}} \prec \frac{1}{d_{1, R}^{1+\gamma_{1}}},
$$

for $\gamma_{1} \geq 0$, and we have:

$$
J\left(E_{2, R}^{1,+}\right)+J\left(E_{2, R}^{1,-}\right)=\int_{E_{2, R}^{1,+} \cup E_{2, R}^{1,-}} \frac{\left|\zeta-z_{1}\right|^{-\gamma_{1}}|d \zeta|}{\left|\zeta-z^{\prime}\right|^{2}} \prec \frac{1}{d_{1, R}},
$$

for $-1<\gamma_{1}<0$. From the inequalities (4.6)-(4.11), we conclude that

$$
\sum_{j=1}^{2}\left(J\left(E_{j, R}^{1,+}\right)+J\left(E_{j, R}^{1,-}\right) \prec \frac{1}{d_{1, R}^{1+\gamma_{1}^{*}}},\right.
$$

where $\tilde{\gamma}_{1}:=\max \left\{\gamma_{1}, 0\right\}$. By the relation (25) and Lemma 2.2 in [4], we write the inequality (4.12) in the following form:

$$
\sum_{j=1}^{2}\left(J\left(E_{j, R}^{1,+}\right)+J\left(E_{j, R}^{1,-}\right) \prec n^{\left(1+\gamma_{1}^{*}\right) \lambda^{*}{ }_{1},}\right.
$$

where

$$
\hat{\lambda}_{1}=\left\{\begin{array}{cc}
\max \left\{\lambda_{1}, 1\right\}, & \text { if } 0<\lambda_{1}<2, \\
2, & \text { if } \lambda_{1}=2 .
\end{array}\right.
$$

Case 2. Let $z^{\prime} \in L^{2}$ and $w^{\prime}=\Phi\left(z^{\prime}\right)$. By changing the variable $\tau=\Phi(\zeta)$ in the integral (4.5), we have:

$$
J_{n} \approx \sum_{i, j=1}^{2} \int_{F_{j, R}^{i, \mid}} \frac{\left|\Psi^{\prime}(\tau)\right||d \tau|}{\Psi(\tau)-\left.\Psi\left(w_{i}\right)\right|^{\gamma_{i}}|\Psi(\tau)-\Psi(w)|^{2}}:=\sum_{i, j=1}^{2} J\left(F_{j, R}^{i, \pm}\right) .
$$

Now, we estimate the integrals $J\left(F_{j, R}^{i, \pm}\right)$ in (4.14). First, we assume that $z^{\prime} \in E_{1}^{2, \pm}$. Then, by the relation (25) and Lemma 2.2 in [4], we have:

$$
\begin{aligned}
J\left(F_{1, R}^{2,+}\right)+J\left(F_{1, R}^{2,-}\right) & =\int_{F_{1, R}^{2,+} \cup F_{1, R}^{2,-}} \frac{\left|\Psi^{\prime}(\tau)\right||d \tau|}{\left|\Psi(\tau)-\Psi\left(w_{2}\right)\right|^{\gamma_{2}}\left|\Psi(\tau)-\Psi\left(w^{\prime}\right)\right|^{2}} \\
& \approx \int_{F_{1, R}^{2,+} \cup F_{1, R}^{2,-}} \frac{d(\Psi(\tau), L)|d \tau|}{\left|\Psi(\tau)-\Psi\left(w_{2}\right)\right|^{\gamma_{2}}\left|\Psi(\tau)-\Psi\left(w^{\prime}\right)\right|^{2}(|\tau|-1)} \\
& \leq \int_{F_{1, R}^{2,} \cup F_{1, R}^{2,-}} \frac{|d \tau|}{d_{2, R}^{\gamma_{2}-1}\left|\Psi(\tau)-\Psi\left(w^{\prime}\right)\right|^{2}(|\tau|-1)} .
\end{aligned}
$$

We estimate only the integral over $F_{1, R}^{2,+}$, since the other estimate is similar. By using the inequality

$$
\left|\Psi(\tau)-\Psi\left(w^{\prime}\right)\right| \geq \min \left\{d_{2, R}, d\left(\Psi(\tau), L^{+}\right)\right\} \succ \min \left\{d_{2, R}, d_{2, R}^{1+\alpha_{2}}\right\} \succ d_{2, R}^{1+\alpha_{2}},
$$

we have 
Ozkartepe / Cumhuriyet Sci. J., Vol.39-1 (2018) 47-65

59

$$
\begin{aligned}
\int_{F_{1, R}^{2,+}} \frac{|d \tau|}{d_{2, R}^{\gamma_{2}-1}\left|\Psi(\tau)-\Psi\left(w^{\prime}\right)\right|^{2}(|\tau|-1)} & \prec \int_{F_{1, R}^{2,+}} \frac{|d \tau|}{d_{2, R}^{\gamma_{2}-1+2\left(1+\alpha_{2}\right)}(|\tau|-1)} \\
& \prec \int_{F_{1, R}^{2,+}} \frac{|d \tau|}{\left|z_{2, R}^{+}-z_{2}^{+}\right|^{\frac{\gamma_{2}-1}{1+\alpha_{2}}+2}(|\tau|-1)} \prec \int_{F_{1, R}^{2,+}} \frac{|d \tau|}{(|\tau|-1)^{\frac{\gamma_{2}-1}{1+\alpha_{2}}+3}} \prec n^{\frac{\gamma_{2}-1}{1+\alpha_{2}+2}} .
\end{aligned}
$$

So that, we get:

$$
J\left(F_{1, R}^{2,+}\right)+J\left(F_{1, R}^{2,-}\right) \prec n^{\frac{\gamma_{2}-1}{1+\alpha_{2}}+2} .
$$

The same conclusion in (4.15) can be drawn for the case $z^{\prime} \in E_{2}^{2, \pm}$, by similar arguments.

Now, assume that $z^{\prime} \in E_{1}^{2, \pm}$. If $\gamma_{2}>0$, then

$$
\begin{aligned}
& J\left(F_{2, R}^{2,+}\right)+J\left(F_{2, R}^{2,-}\right)=\int_{F_{2, R}^{2,+} \cup F_{2, R}^{2,-}} \frac{\left|\Psi^{\prime}(\tau)\right||d \tau|}{\left|\Psi(\tau)-\Psi\left(w_{2}\right)\right|^{\gamma / 2}\left|\Psi(\tau)-\Psi\left(w^{\prime}\right)\right|^{2}} \\
& \prec \int_{F_{2, R}^{2,+}} \frac{|d \tau|}{d_{2, R}^{\gamma_{2}} d(\Psi(\tau), L)^{2}} \prec \int_{F_{2, R}^{2,+R}} \frac{|d \tau|}{\left|z_{2, R}-z_{2}^{+}\right|^{\frac{\gamma 2}{1+\alpha_{2}}}(|\tau|-1)^{2}} \\
& \prec \int_{F_{2, R}^{2,+}} \frac{|d \tau|}{(|\tau|-1)^{\frac{\gamma_{2}}{1+\alpha_{2}}+2}} \prec n^{\frac{\gamma_{2}}{1+\alpha_{2}}+1},
\end{aligned}
$$

and if $-1<\gamma_{2} \leq 0$, then we get:

$$
J\left(F_{2, R}^{2,+}\right)+J\left(F_{2, R}^{2,-}\right) \leq \int_{F_{2, R}^{2,}} \frac{|d \tau|}{d(\Psi(\tau), L)^{2}} \prec \int_{F_{2, R}^{2,+}} \frac{|d \tau|}{(|\tau|-1)^{2}} \prec n .
$$

In the case $z^{\prime} \in E_{2}^{2, \pm}$, if $\gamma_{2}>0$, then we get:

$$
\begin{aligned}
J\left(F_{2, R}^{2,+}\right)+ & J\left(F_{2, R}^{2,-}\right) \prec \int_{F_{2, R}^{2,+}} \frac{|d \tau|}{d_{2, R}^{\gamma_{2}}(|\tau|-1)^{2}}+\int_{F_{2, R}^{2,-}} \frac{|d \tau|}{d_{2, R}^{\gamma_{2}+2}} \\
& \prec \int_{F_{2, R}^{2,+}} \frac{|d \tau|}{\left|z_{2, R}-z_{2}^{+}\right|^{\frac{\gamma_{2}}{1+\alpha_{2}}}(|\tau|-1)^{2}}+\int_{F_{2, R}^{2,-}} \frac{|d \tau|}{\left|z_{2, R}-z_{2}^{+}\right|^{\frac{r^{2}+2}{1+\alpha_{2}}}} \\
& \prec \int_{F_{2, R}^{2,+}} \frac{|d \tau|}{(|\tau|-1)^{\frac{\gamma_{2}}{1+\alpha_{2}}+2}}+\int_{F_{2, R}^{2,-}} \frac{|d \tau|}{(|\tau|-1)^{\frac{\gamma_{2}+2}{1+\alpha_{2}}}} \\
& \prec n^{\frac{\gamma_{2}}{1+\alpha_{2}}+1}+n^{\frac{\gamma_{2}+2}{1+\alpha_{2}}-1}
\end{aligned}
$$

and if $-1<\gamma_{2} \leq 0$, then we have:

$$
J\left(F_{2, R}^{2,+}\right)+J\left(F_{2, R}^{2,-}\right) \prec \int_{F_{2, R}^{2,+}} \frac{|d \tau|}{(|\tau|-1)^{2}}+\int_{F_{2, R}^{2,-}} \frac{|d \tau|}{d_{2, R}^{2}} \prec n+\int_{F_{2, R}^{2,-}} \frac{|d \tau|}{|\tau|-1)^{\frac{2}{1+\alpha_{2}}}} \prec n+n^{\frac{2}{1+\alpha_{2}}-1} .
$$


Consequently, if $z^{\prime}=L^{2}$, we obtain:

$$
J\left(F_{2, R}^{2,+}\right)+J\left(F_{2, R}^{2,-}\right) \prec n^{\frac{\dot{\gamma}_{2}^{*}}{1+\alpha_{2}}+1}
$$

where $\gamma_{2}^{*}=\max \left\{\gamma_{2}, 0\right\}$. If we put the obtained results (4.15) and (4.16) in (4.14), we get the following inequality for the case $z^{\prime} \in L^{2}$ :

$$
J_{n} \prec n^{\frac{\gamma_{2, \alpha_{2}-1}^{*}+\alpha_{2}}{1+\alpha_{2}}}
$$

Where $\gamma_{2, \alpha_{2}}^{*}=\max \left\{\gamma_{2},-\alpha_{2}\right\}$.

For the general case, that is $z^{\prime}=L \quad$, from (4.13) and (4.17), we have:

$$
J_{n} \prec n^{\left(1+\gamma_{1}^{*}\right) \lambda^{*}}+n^{\frac{\gamma_{2}^{*}, \alpha_{2}-1}{1+\alpha_{2}}+2} .
$$

Finally, if we put the estimation (4.18) in (4.3), we obtain the desired result.

\subsection{Proof of Theorem 2.5}

Proof. Suppose that $\operatorname{PDS}\left(\lambda_{1} ; f_{2}\right)$ for some $0<\lambda_{1} \leq 2$ and $f(x)=c x^{1+\alpha_{2}}, \alpha_{2}>0 ; h(z)$ be defined as in (1.1).Throughout this work, we will take $R=1+\frac{1}{n}$. Let $\left\{\xi_{j}\right\}, 1 \leq j \leq m \leq n$, be the zeros (if any) of $P_{n}(z)$ lying on $\Omega$. Lets define the function Blaschke with respect to the zeros $\left\{\xi_{j}\right\}$ of the polynomial $P_{n}(z)$ :

$$
B_{m}(z):=\prod_{j=1}^{m} \frac{\Phi(z)-\Phi\left(\xi_{j}\right)}{1-\overline{\Phi\left(\xi_{j}\right)} \Phi(z)}, z \in \Omega .
$$

It is easy that the $B_{m}\left(\xi_{j}\right)=0,\left|B_{m}(z)\right| \equiv 1$ at $z \in L$ and $\left|B_{m}(z)\right|<1$ at $z \in \Omega$.

For any $p>0$ and $z \in \Omega$ let us set:

$$
T_{n, p}(z):=\left[\frac{P_{n}(z)}{B_{m}(z) \Phi^{n+1}(z)}\right]^{p / 2} .
$$

The function $T_{n, p}(z)$ is analytic in $\Omega$, continuous on $\bar{\Omega}, T_{n, p}(\infty)=0$ and does not have zeros in $\Omega$. We take an arbitrary continuous branch of the $T_{n, p}(z)$ and for this branch, we maintain the same designation. Cauchy integral representation for the unbounded region $\Omega$ gives:

$$
T_{n}(z)=-\frac{1}{2 \pi i} \int_{L} T_{n}(\zeta) \frac{d \zeta}{\zeta-z}, z \in \Omega .
$$

Since $|\Phi(\zeta)|=1$, for $\zeta \in L$, from (4.19) we have: 


$$
\left|P_{n}(z)\right|^{p / 2}=\frac{|\Phi(z)|^{\frac{p(n+1)}{2}}}{2 \pi} \int_{L}\left|P_{n}(\zeta)\right|^{p / 2} \frac{|d \zeta|}{|\zeta-z|} \leq \frac{|\Phi(z)|^{\frac{p(n+1)}{2}}}{2 \pi d(z, L)} \int_{L}\left|P_{n}(\zeta)\right|^{p / 2}|d \zeta| .
$$

Denote by:

$$
A_{n}:=\int_{L}\left|P_{n}(\zeta)\right|^{p / 2}|d \zeta|=\sum_{i=1}^{2} \int_{L^{i}}\left|P_{n}(\zeta)\right|^{p / 2}|d \zeta|
$$

Multiplying the numerator and denominator of the integrand by $h_{0}^{1 / 2}(\zeta) \prod_{j=1}^{2}\left|\zeta-z_{j}\right|^{\gamma_{j} / 2}$, after applying the Holder inequality, from (4.23) we obtain:

$$
\begin{aligned}
A_{n} & \leq \sum_{i=1}^{2}\left(\int_{L^{i}} h(\zeta)\left|P_{n}(\zeta)\right|^{p}|d \zeta|\right)^{1 / 2} \cdot\left(\int_{L^{i}} \frac{|d \zeta|}{\prod_{i=1}^{2}\left|\zeta-z_{i}\right|^{\gamma_{i}}}\right)^{1 / 2} \\
& \prec\left\|P_{n}\right\|_{p}^{p / 2} \cdot\left(\int_{L^{i}} \frac{|d \zeta|}{\left|\zeta-z_{i}\right|^{\gamma_{i}}}\right)^{1 / 2}=:\left\|P_{n}\right\|_{p}^{p / 2} \cdot\left(J_{n}^{1}+J_{n}^{2}\right)^{1 / 2},
\end{aligned}
$$

since the points $z_{1}$ and $z_{2}$ are distinct and where

$$
J_{n}^{i}:=\int_{L^{i}} \frac{|d \zeta|}{\zeta-\left.z_{j}\right|^{\gamma_{j}}}, i=1,2
$$

Taking into consideration notations where we had given in beginning section, estimation (4.24) can be written as following:

$$
A_{n} \prec\left\|P_{n}\right\|_{p}^{p / 2} \cdot\left(J_{n}^{1}+J_{n}^{2}\right)^{1 / 2}=:\left\|P_{n}\right\|_{p}^{p / 2} \sum_{i=1}^{2}\left[I_{n, 1}^{i, \pm}+I_{n, 2}^{i, \pm}\right]^{1 / 2},
$$

where

$$
I_{n, k}^{i, \pm}:=I_{n, k}^{i, \pm}\left(E_{k}^{i, \pm}\right):=\int_{E_{k}^{i, \pm}} \frac{|d \zeta|}{\left|\zeta-z_{i}\right|^{\gamma_{i}}}, \quad i, k=1,2 .
$$

According to (4.22) and (4.23), it is sufficient to estimate the integrals $I_{n, k}^{i, \pm}$ for each $i=1,2$ and $k=1,2$.

Given the possible values of $\gamma_{i}\left(-1<\gamma_{i}<0, \gamma_{i} \geq 0, i=1,2\right)$, we will consider the estimates for the $I_{n, k}^{i, \pm}$ separately.

Let $\gamma_{1} \geq 0$ and $\gamma_{2} \geq 0$. In this case for the integral $J_{n, 2}^{1}$, we get:

$$
I_{n, 1}^{1, \pm}=\int_{E_{1}^{1, \pm}} \frac{|d \zeta|}{\left|\zeta-z_{1}\right|^{\gamma_{1}}}
$$




$$
\begin{gathered}
\prec \int_{0}^{c_{1} d_{1, R}} \frac{d s}{s^{\gamma_{1}}} \prec\left\{\begin{array}{cc}
d_{1, R}^{1-\gamma_{1}}, & \gamma_{1}>1, \\
1, & -1<\gamma_{1} \leq 1 ;
\end{array}\right. \\
I_{n, 2}^{1, \pm}:=\int_{E_{2}^{1, \pm}} \frac{|d \zeta|}{\left|\zeta-z_{1}\right|^{\gamma_{1}}} \prec \int_{c_{1} d_{1, R}}^{\left|l_{1}^{ \pm}\right|} \frac{d s}{s^{\gamma_{1}}} \prec\left\{\begin{array}{cc}
d_{1, R}^{1-\gamma_{1}}, & \gamma_{1}>1, \\
\ln \frac{1}{d_{1, R}}, & \gamma_{1}=1, \\
1, & -1<\gamma_{1}<1 .
\end{array}\right.
\end{gathered}
$$

Similar estimate for the integral $J_{n}^{2}$ :

$$
\begin{gathered}
I_{n, 1}^{2, \pm}=\int_{E_{1}^{2, \pm}} \frac{|d \zeta|}{\left|\zeta-z_{2}\right|^{\gamma_{2}}} \prec \int_{0}^{c_{2} d_{2, R}} \frac{d s}{s^{\gamma_{2}}} \prec\left\{\begin{array}{cc}
d_{2, R}^{1-\gamma_{2}}, & \gamma_{2}>1, \\
1, & -1<\gamma_{2} \leq 1 ;
\end{array}\right. \\
I_{n, 2}^{2, \pm}=\int_{E_{2}^{2, \pm}} \frac{|d \zeta|}{\left|\zeta-z_{2}\right|^{\gamma_{2}}} \prec \int_{c_{2} d_{2, R}}^{\left|l_{2}^{ \pm}\right|} \frac{d s}{s^{\gamma_{2}}} \prec\left\{\begin{array}{cc}
d_{2, R}^{1-\gamma_{2}}, & \gamma_{2}>1, \\
\ln \frac{1}{d_{2, R},} & \gamma_{2}=1, \\
1, & -1<\gamma_{2}<1 .
\end{array}\right.
\end{gathered}
$$

Let $\gamma_{1}<0$ and $\gamma_{2}<0$. Then, analogously to the (4.27) and (4.28), we get:

$$
\begin{gathered}
I_{n, 1}^{1, \pm}=\int_{E_{1}^{1, \pm}}\left|\zeta-z_{1}\right|^{\left(-\gamma_{1}\right)}|d \zeta| \prec d_{1, n}^{\left(-\gamma_{1}\right)} \operatorname{mes} E_{1}^{1} \prec 1, \\
I_{n, 2}^{1, \pm}=\int_{E_{2}^{1, \pm}}\left|\zeta-z_{1}\right|^{\left(-\gamma_{1}\right)}|d \zeta| \prec\left|l_{1}^{ \pm}\right|^{1-\gamma_{1}} \prec 1 ; \\
I_{n, 1}^{2, \pm} \prec \int_{E_{1}^{2, \pm}}\left|\zeta-z_{2}\right|^{\left(-\gamma_{2}\right)}|d \zeta| \prec d_{2, R}^{\left(-\gamma_{2}\right)} m e s E_{1}^{2, \pm} \prec 1, \\
I_{n, 2}^{2, \pm} \prec \int_{E_{2}^{2, \pm}}\left|\zeta-z_{2}\right|^{\left(-\gamma_{2}\right)}|d \zeta| \prec\left|l_{2}^{ \pm}\right|^{1-\gamma_{2}} \prec 1 .
\end{gathered}
$$

Therefore, in this case, from (4.26) - (4.30), we obtain:

$$
A_{n} \prec\left\|P_{n}\right\|_{p}^{p / 2} \cdot\left\{\begin{array}{cc}
d_{1, R}^{\frac{1-\gamma_{1}}{2}}+d_{2, R}^{\frac{1-\gamma_{2}}{2}}, & \gamma_{1}, \gamma_{2}>1, \\
{\left[\ln \frac{1}{d_{1, R}}+\ln \frac{1}{d_{2, R}}\right]^{\frac{1}{2}},} & \gamma_{1}=\gamma_{2}=1, \\
1, & -1<\gamma_{1}, \gamma_{2}<1 .
\end{array}\right.
$$

Comparing (4.22), (4.23) and (4.31), we have:

$$
\left|P_{n}(z)\right| \leq c \frac{\left(A_{n, 1}^{0}\right)^{1 / p}}{d^{p / 2}(z, L)}\left\|P_{n}\right\|_{p}|\Phi(z)|^{n+1},
$$


where $c=c\left(G, p, \gamma_{i}\right)>0$, is the constant independent from $n$ and $z$, and

$$
A_{n, 1}^{0}:=\left\{\begin{array}{cc}
d_{1, R}^{1-\gamma_{1}}+d_{2, R}^{1-\gamma_{2}}, & \gamma_{1}, \gamma_{2}>1, \\
\ln \frac{1}{d_{1, R}}+\ln \frac{1}{d_{2, R}}, & \gamma_{1}=\gamma_{2}=1, \\
1, & -1<\gamma_{1}, \gamma_{2}<1 .
\end{array}\right.
$$

According to [8, Lemma 1.1] and Lemma 3.2, for the point $z_{1}$ we get

$$
d_{1, R} \succ n^{-\lambda_{1}^{*}}
$$

For the estimate $d_{2, R}$, let's set: $z_{2, R} \in L_{R}$ such that $d_{2, R}=\left|z_{2}-z_{2, R}\right| ; \zeta^{ \pm} \in L^{ \pm} \quad$ such that $d\left(z_{2, R}, L^{2} \cap L^{ \pm}\right):=d\left(z_{2, R}, L^{+}\right) ; z_{2}^{ \pm}:=\zeta \in L^{2}:\left|\zeta-z_{2}\right|=c_{2} d_{2, R}$. Under these notations, from Lemma 3.1, we obtain:

$$
d_{R}^{ \pm}:=d\left(z_{2, R}, L^{2} \cap L^{ \pm}\right) \approx\left|z_{2, R}-z_{2}^{ \pm}\right| \approx d_{2, R}^{1+\alpha}
$$

Hence, $d_{2, R}=\left(d_{R}^{ \pm}\right)^{\frac{1}{1+\alpha}}$. On the other hand, according to Lemma 3.2 and [8, Lemma 1.1], we get: $d_{R}^{ \pm} \succ \frac{1}{n}$. Therefore,

$$
d_{2, R} \succ n^{\frac{-1}{1+\alpha}} .
$$

Comparing (4.32)-(4.36), we get:

$$
\left|P_{n}(z)\right| \prec \frac{A_{n, 1}}{d^{p / 2}(z, L)}\left\|P_{n}\right\|_{p}|\Phi(z)|^{n+1}, z \in \Omega,
$$

where

$$
A_{n, 1}:=\left\{\begin{array}{cc}
n^{\frac{\left(\gamma_{1}-1\right) \lambda^{*}}{p}}+n^{\frac{\gamma_{2}-1}{p(1+\alpha)}}, & \gamma_{1}, \gamma_{2}>1, \\
(\ln n)^{\frac{1}{p}}, & \gamma_{1}=\gamma_{2}=1, \\
1, & -1<\gamma_{1}, \gamma_{2}<1,
\end{array}\right.
$$

and we complete the proof .

The proof of Theorems 2.2, 2.3 and 2.4 it is not difficult to obtain from the scheme of the proof of Theorem 2.1. 


\section{REFERENCES}

[1] Abdullayev F.G., Andrievskii V.V. On the orthogonal polynomials in the domains with $K$ quasiconformal boundary. Izv. Akad. Nauk Azerb. SSR., Ser. FTM 1983; 1: 3-7.

[2] Abdullayev F. G., Özkartepe N. P., Gün C. D. Uniform and pointwise polynomial inequalities in regions without cusps in the weighted Lebesgue space. Bulletin of Tbilisi ICMC , 18-1 (2014) 146167.

[3] Abdullayev F.G., Özkartepe P. On the growth of algebraic polynomials in the whole complex plane. J. Korean Math. Soc., 52-4 (2015) 699-725.

[4] Abdullayev F. G., Gün C.D., Ozkartepe N.P. Inequalities for algebraic polynomials in regions with exterior cusps. J. Nonlinear Funct. Anal., Article ID 3 (2015) 1-32.

[5] Abdullayev F.G., Özkartepe P. Uniform and pointwise polynomial inequalities in regions with cusps in the weighted Lebesgue space. Jaen Journal on Approximation, 7-2 (2015) 231-261.

[6] Abdullayev F.G., Özkartepe P., Polynomial inequalities in Lavrentiev regions with interior and exterior zero angles in the weighted Lebesgue space. Publications de l'Institut Mathématique (Beograd), 100-114 (2016) 209-227.

[7] Ahlfors L. Lectures on Quasiconformal Mappings. Princeton, NJ: Van Nostrand, 1966.

[8] Andrievskii V.V. On the uniform convergence of the Bieberbach polynomials in the regions with piecewise quasiconformal boundary, In: Theory of Mappings and approximation functions, "Naukovo Dumka", Kyiv, (1983) 3-18. (in Russian)

[9] Andrievskii V.V., Weighted Polynomial Inequalities in the Complex Plane. Journal of Approximation Theory, 164-9 (2012) 1165-1183.

[10] Andrievskii V.V., Belyi V.I. \& Dzyadyk V.K. Conformal invariants in constructive theory of functions of complex plane. Atlanta:World Federation Publ.Com., 1995.

[11] Andrievskii V.V., Blatt H.P. Discrepancy of Signed Measures and Polynomial Approximation, Springer Verlag New York Inc., 2010.

[12] Dzjadyk V.K., Introduction to the Theory of Uniform Approximation of Function by Polynomials, Nauka, Moskow, 1977. (in Russian)

[13] Gaier D., On the convergence of the Bieberbach polynomials in regions with corners. Constructive Approximation, 4 (1988) 289-305.

[14] Jackson D., Certain problems on closest approximations. Bull. Amer. Math. Soc., 39 (1933) 889906.

[15] Lehto O., Virtanen K.I., Quasiconformal Mapping in the plane, Springer Verlag, Berlin, 1973.

[16] Mamedhanov D.I., Inequalities of S.M.Nikol'skii type for polynomials in the complex variable on curves, Soviet Math.Dokl., 15 (1974) 34-37.

[17] Mamedhanov D.I., On Nikol'skii-type inequalities with new characteristics, Doklady Mathematics, 82 (2010) 882-883.

[18] Milovanovic G.V., Mitrinovic D.S. and Rassias Th.M., Topics in Polynomials: Extremal Problems, Inequalities, Zeros, World Scientific, Singapore, 1994.

[19] Nikol'skii S.M., Approximation of function of several variable and imbeding theorems, SpringerVerlag, New-York, 1975.

[20] Özkartepe N. P., Abdullayev F. G. , On the interference of the weight and boundary contour for algebraic polynomials in the weighted Lebesgue spaces I. Ukr. Math. J. 2016; 68(10): (Trans. from Ukr. Mat. Zh., 68-10 (2016) 1365-1379.

[21] Pritsker I.E., Comparing norms of polynomials in one and several variables, Journal of Math. Anal. 
and Appl., 216 (1997) 685-695.

[22] Pommerenke Ch., Univalent Functions, Göttingen, Vandenhoeck \& Ruprecht, 1975.

[23] Rickman S., Characterization of quasiconformal arcs. Ann. Acad. Sci. Fenn., Ser. A, Mathematica, 395 (1966) $30 \mathrm{p}$.

[24] Stylianopoulos N., Strong asymptotics for Bergman polynomials over domains with corners and applications. Const. Approx., 38 (2013) 59-100.

[25] Suetin P.K., The ordinally comparison of various norms of polynomials in the complex domain, Matematicheskie zapiski Uralskogo Gos. Universiteta, $5-4$ (1966) (in Russian).

[26] Suetin P. K., Main properties of the orthogonal polynomials along a circle, Uspekhi Math. Nauk, 21-2,128 (1966) 41-88.

[27] Suetin P.K., On some estimates of the orthogonal polynomials with singularities weight and contour. Sibirskiy Mat. Zhurnal, VIII-3 (1967) 1070-1078 (in Russian).

[28] Szegö G., Zygmund A., On certain mean values of polynomials. Journal d'Analyse Mathematique, 3-1 (1953) 225-244.

[29] Walsh J.L., Interpolation and Approximation by Rational Functions in the Complex Domain, AMS, 1960. 